\title{
EXPLICIT SOLUTION FORMULAS FOR THE MATRIX-KP
}

\author{
CORNELIA SCHIEBOLD \\ Department of Mathematics, Mid Sweden University, S-851 70 Sundsvall, Sweden \\ e-mail: Cornelia.Schiebold@miun.se
}

\begin{abstract}
We study a non-commutative version of the Kadomtsev-Petviashvili equations and construct a family of solutions generalizing naturally the soliton to the non-commutative setting. From this we derive explicit solution formulas as well for the scalar as for the matrix-Kadomtsev-Petviashvili equation which still depend on operator parameters.
\end{abstract}

2000 Mathematics Subject Classification. 39B42, 47J25, 37K10, 35C05.

1. Introduction and main results. The first aim of the present paper is to find a general solution formula for the non-commutative Kadomtsev-Petviashvili equations (ncKP)

$$
\left(U_{t}-6\left(U_{x}\right)^{2}+U_{x x x}\right)_{x}+3 \alpha^{2} U_{y y}-6 \alpha\left[U_{x}, U_{y}\right]=0,
$$

with $\alpha=\mathrm{i}$ for the KP-I, $\alpha=1$ for the KP-II. The unknown function $U=U(x, y, t)$ is supposed to take values in the space $\mathcal{L}(F)$ of continuous linear operators on some Banach space $F$. This includes the matrix-KP equations for $\operatorname{dim} F<\infty$. Noncommutative integrable systems attracted much interest recently (cf. for example $[2,8,9,13,14]$. Particularly close in spirit to the present paper are $[5-7,10])$.

Our result on the general operator level is the following:

THEOREM 1.1. Let $E$ and $F$ be Banach spaces and $A \in \mathcal{L}(E), B \in \mathcal{L}(F)$.

Assume that $L=L(x, y, t) \in \mathcal{L}(F, E), \quad M=M(x, y, t) \in \mathcal{L}(E, F)$ are operatorfunctions which are $C^{4}$-smooth and solve the base equations

$$
\begin{aligned}
& L_{x}=A L, \quad L_{y}=\alpha^{-1} A^{2} L, \quad L_{t}=-4 A^{3} L, \\
& M_{x}=B M, \quad M_{y}=-\alpha^{-1} B^{2} M, \quad M_{t}=-4 B^{3} M \text {. }
\end{aligned}
$$

Then, on $\Omega=\left\{(x, y, t) \in \mathbb{R}^{3} \mid(I+M L)\right.$ is invertible $\}$, a solution of the $n c K P(1)$ with values in the algebra $\mathcal{L}(F)$ is given by

$$
U=(I+M L)^{-1}(B-M A L) .
$$

In [3] the result was obtained under the additional assumption $[A, B]=0$ (see also [11]), which is very restrictive in applications. One of our motivations were similar formulas as of Sakhnovich [10], which were only verified by means of computer algebra. In fact our proof of Theorem 1.1 (improving the original argument in [12]) still requires considerable calculations. It is given in Section 2.

Our second aim, crucial in view of applications, is to extract solution formulas for the matrix-KPs (including the scalar case). The point is of course to obtain for a given matrix equation a more powerful formula than the one already provided 
by Theorem 1.1. Actually we will find formulas depending effectively on three free parameters each of which is an operator between Banach spaces. A fourth operator has to be chosen appropriately in some quasi-Banach ideal to ensure existence of certain determinants. Here, it is profitable to use the generality of Banach ideals: we refer to [1] (see also [4]) where it is shown that for the Korteweg-de Vries equation $(\mathrm{KdV})$ all solutions of the standard Inverse Scattering Method (ISM) are accessible via a comparable ncKdV theory.

Our second main result is the following:

THeOrem 1.2. Let $E$ and $F$ be Banach spaces and $A \in \mathcal{L}(E), B \in \mathcal{L}(F)$.

Assume that there is an operator $C \in \mathcal{A}(F, E)$ belonging to a quasi-Banach ideal $\mathcal{A}$ admitting a continuous determinant $\delta$, which satisfies the $n$-dimensionality condition

$$
A C+C B=\sum_{j=1}^{n} a_{j} \otimes c_{j}
$$

with linearly independent functionals $a_{j} \in F^{\prime}$ and vectors $c_{j} \in E$ for $j=1, \ldots, n$.

Moreover, let $D \in \mathcal{L}(E, F)$ arbitrarily.

Then, provided the denominator does not vanish, a solution of the matrix-KP (1) with values in the $n \times n$-matrices is given by

$$
u=\left(1-\frac{\delta\left(I+M L+a_{i} \otimes\left(M \ell_{j}\right)\right)}{\delta(I+M L)}\right)_{i, j=1}^{n}
$$

with the operator-functions $L=\hat{L} C, M=\hat{M} D$, the vector-functions $\ell_{j}=\hat{L} c_{j}$ for $j=1, \ldots, n$, where $\hat{L}(x, y, t)=\exp \left(A x+\alpha^{-1} A^{2} y-4 A^{3} t\right)$ and $\hat{M}(x, y, t)=\exp (B x-$ $\left.\alpha^{-1} B^{2} y-4 B^{3} t\right)$.

The above solution formula depends effectively on the parameters $A, B$ and $D$. Note that the condition (3) can be satisfied under a mild spectral condition on $A$ and $B$ (cf. [12]). Furthermore it is interesting that Theorem 1.2 also establishes links between matrix equations of different dimensions. Theorem 1.2 is proved in Section 3 . A noteworthy simplification for the scalar case is explained in Section 4.

For lack of space we did not include examples. The interested reader finds some material related to Miles structures in the author's habilitation thesis [12]. Applications are planned to be the topic of a forthcoming publication.

2. The soliton solution of the ncKP equations. In this section we prove Theorem 1.1. To this end, we consider the following situation: Let $G$ be a Banach space and $J \in \mathcal{L}(G)$ an involution (i.e. $J^{2}=I$ ). For an operator-valued function $S=S\left(x_{1}, x_{2}, x_{3}\right) \in \mathcal{L}(G)$ we consider the non-commutative operator equation

$$
\left(S_{x_{1} x_{1} x_{1}}-6\left(S_{x_{1}}\right)^{2}-4 S_{x_{3}}\right)_{x_{1}}=6 J\left[S_{x_{2}}, S_{x_{1}}\right]-3 S_{x_{2} x_{2}}
$$

a non-commutative version of the KP depending on the involution $J$. We will obtain Theorem 1.1 as a consequence of Theorem 2.1.

Theorem 2.1. Let $C \in \mathcal{L}(G)$ where $[C, J]=0$. Assume that $N=N(x, t) \in \mathcal{L}(G)$ is an operator-valued function anti-commuting with $J$ and solving the base equations 
$N_{x_{1}}=C N, N_{x_{2}}=J C^{2} N$ and $N_{x_{1}}=C^{3} N$. Then

$$
S=\left(I-N^{2}\right)^{-1}(C+N C N)
$$

solves the operator equation (4) wherever $\left(I-N^{2}\right)$ is invertible.

For the proof we introduce for $i \in \mathbb{N}$ the operators $C_{i}=J(J C)^{i}$ (The base equations now read $N_{x_{i}}=C_{i} N$ for $\left.i=1,2,3\right)$ and the operator-functions

$$
\begin{aligned}
& S_{i}=\left(I-N^{2}\right)^{-1}\left(C_{i}+N C_{i} N\right), \\
& T_{i}=\left(I-N^{2}\right)^{-1}\left(C_{i} N+N C_{i}\right) .
\end{aligned}
$$

The next two lemmas furnish the recursive identities which allow to cut down the proof of Theorem 2.1 to a reasonable size.

Lemma 2.2. The derivative of the operator-functions $S_{i}=S_{i}\left(x_{1}, x_{2}, x_{3}\right), T_{i}=$ $T_{i}\left(x_{1}, x_{2}, x_{3}\right)$ given in (5) and (6) with respect to $x_{j}, j=1,2,3$, is

$$
S_{i, x_{j}}=T_{j} T_{i}, \quad T_{i, x_{j}}=S_{j} T_{i} .
$$

Proof. Recall that for a differentiable function $R=R(\xi)$ with values in the invertible operators we have $\left(R^{-1}\right)_{\xi}=-R^{-1} R_{\xi} R^{-1}$. Using the base equations and $\left[C_{i}, C_{j}\right]=0$ for all $i, j$, we infer

$$
\begin{aligned}
T_{i, x_{j}} & =-\left(I-N^{2}\right)^{-1}\left(-N^{2}\right)_{x_{j}}\left(I-N^{2}\right)^{-1}\left(C_{i} N+N C_{i}\right)+\left(I-N^{2}\right)^{-1}\left(C_{i} N+N C_{i}\right)_{x_{j}} \\
& =\left(I-N^{2}\right)^{-1}\left(\left(C_{j} N+N C_{j}\right) N\right)\left(I-N^{2}\right)^{-1}\left(C_{i} N+N C_{i}\right)+\left(I-N^{2}\right)^{-1} C_{j}\left(C_{i} N+N C_{i}\right) \\
& =\left(I-N^{2}\right)^{-1}\left(\left(C_{j} N+N C_{j}\right) N+C_{j}\left(I-N^{2}\right)\right) T_{i} \\
& =\left(I-N^{2}\right)^{-1}\left(C_{j}+N C_{j} N\right) T_{i}=S_{j} T_{i}, \\
S_{i, x_{j}} & =-\left(I-N^{2}\right)^{-1}\left(-N^{2}\right)_{x_{j}}\left(I-N^{2}\right)^{-1}\left(C_{i}+N C_{i} N\right)+\left(I-N^{2}\right)^{-1}\left(C_{i}+N C_{i} N\right)_{x_{j}} \\
& =\left(I-N^{2}\right)^{-1}\left(\left(C_{j} N+N C_{j}\right) N\right)\left(I-N^{2}\right)^{-1}\left(C_{i}+N C_{i} N\right)+\left(I-N^{2}\right)^{-1}\left(C_{j} N+N C_{j}\right) C_{i} N \\
& =T_{j}\left(I-N^{2}\right)^{-1}\left(N\left(C_{i}+N C_{i} N\right)+\left(I-N^{2}\right) C_{i} N\right) \\
& =T_{j}\left(I-N^{2}\right)^{-1}\left(C_{i} N+N C_{i}\right)=T_{j} T_{i} .
\end{aligned}
$$

LEMMA 2.3. For the operator-functions $S_{i}$ and $T_{i}$ given in (5) and (6), the following identities hold:

$$
S_{i} T_{j}-T_{i} S_{j}=J T_{i+j}, \quad S_{i} S_{j}-T_{i} T_{j}=J S_{i+j}
$$

Proof. We need the following auxiliary identity:

$$
N\left(I-N^{2}\right)^{-1} N=\left(I-N^{2}\right)^{-1} N^{2}=\left(I-N^{2}\right)^{-1}\left(I-\left(I-N^{2}\right)\right)=\left(I-N^{2}\right)^{-1}-I,
$$

which is applied in the third step of the succeeding calculation to replace the terms in the first and in the last large brackets.

$$
\begin{aligned}
\left(I-N^{2}\right) S_{i} T_{j}= & \left(C_{i}+N C_{i} N\right)\left(I-N^{2}\right)^{-1}\left(C_{j} N+N C_{j}\right) \\
= & C_{i}\left(\left(I-N^{2}\right)^{-1}\right) C_{j} N+C_{i}\left(\left(I-N^{2}\right)^{-1} N\right) C_{j} \\
& +N C_{i}\left(N\left(I-N^{2}\right)^{-1}\right) C_{j} N+N C_{i}\left(N\left(I-N^{2}\right)^{-1} N\right) C_{j}
\end{aligned}
$$




$$
\begin{aligned}
= & C_{i}\left(I+N\left(I-N^{2}\right)^{-1} N\right) C_{j} N+C_{i}\left(N\left(I-N^{2}\right)^{-1}\right) C_{j} \\
& +N C_{i}\left(\left(I-N^{2}\right)^{-1} N\right) C_{j} N+N C_{i}\left(\left(I-N^{2}\right)^{-1}-I\right) C_{j} \\
= & \left(C_{i} C_{j} N-N C_{i} C_{j}\right)+\left(C_{i} N+N C_{i}\right)\left(I-N^{2}\right)^{-1}\left(C_{j}+N C_{j} N\right) \\
= & J\left(C_{i+j} N+N C_{i+j}\right)+\left(I-N^{2}\right) T_{i} S_{j},
\end{aligned}
$$

where for the last step we have used $[J, C]=0$ to have $C_{i} C_{j}=J(J C)^{i} J(J C)^{j}=$ $(J C)^{i+j}=J C_{i+j}$, and also $\{J, N\}=0$. Similarly,

$$
\begin{aligned}
\left(I-N^{2}\right) S_{i} S_{j}= & \left(C_{i}+N C_{i} N\right)\left(I-N^{2}\right)^{-1}\left(C_{j}+N C_{j} N\right) \\
= & C_{i}\left(\left(I-N^{2}\right)^{-1}\right) C_{j}+C_{i}\left(\left(I-N^{2}\right)^{-1} N\right) C_{j} N \\
& +N C_{i}\left(N\left(I-N^{2}\right)^{-1}\right) C_{j}+N C_{i}\left(N\left(I-N^{2}\right)^{-1} N\right) C_{j} N \\
= & C_{i}\left(I+N\left(I-N^{2}\right)^{-1} N\right) C_{j}+C_{i}\left(N\left(I-N^{2}\right)^{-1}\right) C_{j} N \\
& +N C_{i}\left(\left(I-N^{2}\right)^{-1} N\right) C_{j}+N C_{i}\left(\left(I-N^{2}\right)^{-1}-I\right) C_{j} N \\
= & \left(C_{i} C_{j}-N C_{i} C_{j} N\right)+\left(C_{i} N+N C_{i}\right)\left(I-N^{2}\right)^{-1}\left(C_{j} N+N C_{j}\right) \\
= & J\left(C_{i+j}+N C_{i+j} N\right)+\left(I-N^{2}\right) T_{i} T_{j},
\end{aligned}
$$

Proof of Theorem 2.1. To simplify notation, we write $S=S_{1}, T=T_{1}$. Observe also that the operator-functions $S_{i}$ commute with the involution $J$ whereas the $T_{i}$ anti-commute with $J$.

We start by deriving two auxiliary identities. By Lemmas 2.2 and 2.3 applied to the respective products in the brackets, we infer

$$
\begin{aligned}
\left(T_{j} T\right)_{x_{k}} & =T_{j, x_{k}} T+T_{j} T_{x_{k}}=\left(S_{k} T_{j}\right) T+T_{j} S_{k} T \\
& =J T_{j+k} T+\left(T_{k} S_{j}+T_{j} S_{k}\right) T \\
\left(T_{i} S_{j} T\right)_{x_{1}} & =T_{i, x_{1}} S_{j} T+T_{i} S_{j, x_{1}} T+T_{i} S_{j} T_{x_{1}} \\
& =\left(S T_{i}\right) S_{j} T+T_{i} T T_{j} T+T_{i}\left(S_{j} S\right) T \\
& =\left(J T_{i+1}+T S_{i}\right) S_{j} T+T_{i} T T_{j} T+T_{i}\left(J S_{j+1}+T_{j} T\right) T \\
& =J\left(T_{i+1} S_{j}-T_{i} S_{j+1}\right) T+\left(T\left(S_{i} S_{j}\right)+T_{i} T T_{j}+T_{i} T_{j} T\right) T \\
& =J\left(T_{i+1} S_{j}-T_{i} S_{j+1}-T S_{i+j}\right) T+\left(T T_{i} T_{j}+T_{i} T T_{j}+T_{i} T_{j} T\right) T
\end{aligned}
$$

for all $i, j \in \mathbb{N}$ and $k=1,2,3$. Now,

$$
S_{x_{1}}=T^{2} \quad \text { and } \quad S_{x_{2}}=T_{2} T, \quad S_{x_{3}}=T_{3} T
$$

by Lemma 2.2. From (7) with $j=k=1$ we get $S_{x_{1} x_{1}}=J T_{2} T+2 T S T$. Applying (7) with $j=2, k=1$ and (8) with $i=j=1$ then yields

$$
\begin{aligned}
S_{x_{1} x_{1} x_{1}} & =J\left(T_{2} T\right)_{x_{1}}+2(T S T)_{x_{1}} \\
& =J\left(J T_{3} T+\left(T S_{2}+T_{2} S\right) T\right)+2\left(J\left(T_{2} S-2 T S_{2}\right) T+3 T^{4}\right) \\
& =T_{3} T+3 J\left(T_{2} S T-T S_{2} T\right)+6 T^{4} .
\end{aligned}
$$

Together with (9) this results in

$$
S_{x_{1} x_{1} x_{1}}-6\left(S_{x_{1}}\right)^{2}-4 S_{x_{3}}=-3 T_{3} T+3 J\left(T_{2} S T-T S_{2} T\right) .
$$


Before taking the derivative with respect to $x_{1}$ once more, we need another auxiliary identity. First we remark that, by (7) and (9) with $j=k=2$,

$$
\left[S_{x_{2}}, S_{x_{1}}\right]=\left[T_{2}, T^{2}\right] T, \quad S_{x_{2} x_{2}}=\left(T_{2} T\right)_{x_{2}}=J T_{4} T+2 T_{2} S_{2} T .
$$

Thus, using (8) both with $i=2, j=1$ and $i=1, j=2$, we find

$$
\begin{aligned}
\left(T_{2} S T-T S_{2} T\right)_{x_{1}} & =J\left(T S_{3}+T_{3} S\right) T-2 J T_{2} S_{2} T+2\left(T_{2} T^{2}-T^{2} T_{2}\right) T \\
& =J\left(T S_{3}+T_{3} S\right) T+T_{4} T-J S_{x_{2} x_{2}}+2\left[S_{x_{2}}, S_{x_{1}}\right] .
\end{aligned}
$$

Now we use (7) and the latter identity to finally verify

$$
\begin{aligned}
\left(S_{x_{1} x_{1} x_{1}}-6\left(S_{x_{1}}\right)^{2}-4 S_{x_{3}}\right)_{x_{1}}= & -3\left(T_{3} T\right)_{x_{1}}+3 J\left(T_{2} S T-T S_{2} T\right)_{x_{1}} \\
= & -3\left(J T_{4} T+\left(T S_{3}+T_{3} S\right) T\right)+3 J\left(J\left(T S_{3}+T_{3} S\right) T\right. \\
& \left.+T_{4} T-J S_{x_{2} x_{2}}+2\left[S_{x_{2}}, S_{x_{1}}\right]\right) \\
= & -3 S_{x_{2} x_{2}}+6 J\left[S_{x_{2}}, S_{x_{1}}\right] .
\end{aligned}
$$

Proof of Theorem 1.1. Introducing the coordinate transformation $x_{1}=x, x_{2}=$ $\alpha^{-1} y$ and $x_{3}=-4 t$, the base equations become $L_{x_{j}}=A^{j} L$ and $M_{x_{j}}=-(-B)^{j} M$ for $j=1,2,3$.

Now it is immediate that the requirements of Theorem 2.1 are met with the following choices:

$$
G:=E \oplus F, \quad J:=\left(\begin{array}{cc}
I & 0 \\
0 & -I
\end{array}\right), \quad C:=\left(\begin{array}{cc}
A & 0 \\
0 & B
\end{array}\right), \quad N:=\left(\begin{array}{cc}
0 & -L \\
M & 0
\end{array}\right) .
$$

Hence,

$$
\begin{aligned}
S & =\left(I-N^{2}\right)^{-1}(C+N C N) \\
& =\left(\begin{array}{cc}
I+L M & 0 \\
0 & I+M L
\end{array}\right)^{-1}\left(\begin{array}{cc}
A-L B M & 0 \\
0 & B-M A L
\end{array}\right) \\
& =\left(\begin{array}{cc}
(I+L M)^{-1}(A-L B M) & 0 \\
0 & (I+M L)^{-1}(B-M A L)
\end{array}\right)
\end{aligned}
$$

solves (4). Since (4) in turn splits up in its diagonal parts, from the part in the lower left corner reading

$$
\left(U_{x_{1} x_{1} x_{1}}-6\left(U_{x_{1}}\right)^{2}-4 U_{x_{3}}\right)_{x_{1}}=6\left[U_{x_{1}}, U_{x_{2}}\right]-3 U_{x_{2} x_{2}}
$$

we can conclude that $U=(I+M L)^{-1}(B-M A L)$, the lower left corner of the above operator-function $S$, solves (10). It remains to observe that (10) transforms to (1) under the coordinate change $x_{1}=x, x_{2}=\alpha^{-1} y$ and $x_{3}=-4 t$.

REMARK 2.4. Focusing on the upper right corner, we again get a solution of (10) but with a minus sign in front of the commutator. Transforming $y$ into $-y$, again a solution for (1) is obtained. The latter corresponds to Theorem 1.1 with the roles of $A$ and $B$ interchanged. 
3. Solution formulas for the matrix-KP equations. In this section the soliton solution (2) of the ncKP is used to construct an explicit solution formula for the matrix-KP. The idea is to descend via a continuous, linear map $\sigma$ from $\mathcal{L}(F)$ to the space of $n \times n$-matrices. To guarantee that a solution $U=U(x, y, t) \in \mathcal{L}(F)$ transforms to a matrix solution $u=\sigma(U)$, we will require $\sigma$ to be multiplicative in a suitable sense.

3.1. Algebraic tools. Let $F$ be a Banach space. Recall that a one-dimensional endomorphism on $F$ can be written as $a \otimes y$ with appropriate $a \in F^{\prime}, y \in F$ where the map $a \otimes y$ is defined by $(a \otimes y)(v)=\langle v, a\rangle y$ (and $\langle v, a\rangle$ denotes the evaluation of the functional $a$ on $v \in F$ ).

For linearly independent functionals $a_{1}, \ldots, a_{n} \in F^{\prime}$, we define the vector space

$$
\mathcal{S}_{a_{1}, \ldots, a_{n}}(F)=\left\{\sum_{j=1}^{n} a_{j} \otimes y_{j} \mid y_{1}, \ldots, y_{n} \in F\right\} .
$$

Equivalently this is the space of all endomorphisms $T$ satisfying $\operatorname{ker}(T) \supset \bigcap_{j=1}^{n} \operatorname{ker}\left(a_{j}\right)$. Note that every such $T$ has rank at most $n$ and possesses a unique representation $T=\sum_{j=1}^{n} a_{j} \otimes y_{j}$ with $y_{1}, \ldots, y_{n} \in F$.

LEMMA 3.1. $\mathcal{S}_{a_{1}, \ldots, a_{n}}(F)$ is a Banach algebra and a left ideal in $\mathcal{L}(F)$.

For given $a_{1}, \ldots, a_{n}$, we define the (continuous, linear) map $\sigma$ from $\mathcal{S}_{a_{1}, \ldots, a_{n}}(E)$ to the space of $n \times n$-matrices with complex entries by

$$
\sigma\left(\sum_{j=1}^{n} a_{j} \otimes y_{j}\right)=\left(\left\langle y_{j}, a_{i}\right\rangle\right)_{i, j=1}^{n} .
$$

Of course $\sigma=\sigma_{a_{1}, \ldots, a_{n}}$. In the sequel we do not mention the dependence on the functionals $a_{1}, \ldots, a_{n}$ if it is clear from the context.

Proposition 3.2. On $\mathcal{S}_{a_{1}, \ldots, a_{n}}(F)$, the map $\sigma$ given by (11) is an algebra homomorphism.

Proof. First observe that one-dimensional operators are multiplied via $(a \otimes y)$ $(b \otimes z)=\langle z, a\rangle b \otimes y$ for $a, b \in F^{\prime}$ and $y, z \in F$. Now let $T, S \in \mathcal{S}_{a_{1}, \ldots, a_{n}}(F)$, say $T=$ $\sum_{j=1}^{n} a_{j} \otimes y_{j}, S=\sum_{j=1}^{n} a_{j} \otimes z_{j}$ where $y_{j}, z_{j} \in F$ for $j=1, \ldots, n$. Then,

$$
\begin{aligned}
\sigma(T S) & =\sigma\left(\sum_{k=1}^{n} a_{k} \otimes y_{k} \cdot \sum_{j=1}^{n} a_{j} \otimes z_{j}\right)=\sigma\left(\sum_{j=1}^{n} a_{j} \otimes\left(\sum_{k=1}^{n}\left\langle z_{j}, a_{k}\right\rangle y_{k}\right)\right) \\
& =\left(\left\langle\sum_{k=1}^{n}\left\langle z_{j}, a_{k}\right\rangle y_{k}, a_{i}\right\rangle\right)_{i, j=1}^{n}=\left(\sum_{k=1}^{n}\left\langle y_{k}, a_{i}\right\rangle\left\langle z_{j}, a_{k}\right\rangle\right)_{i, j=1}^{n} \\
& =\left(\left\langle y_{j}, a_{i}\right\rangle\right)_{i, j=1}^{n}\left(\left\langle z_{j}, a_{i}\right\rangle\right)_{i, j=1}^{n}=\sigma\left(\sum_{j=1}^{n} a_{j} \otimes y_{j}\right) \sigma\left(\sum_{j=1}^{n} a_{j} \otimes z_{j}\right)=\sigma(T) \sigma(S) .
\end{aligned}
$$

REMARK 3.3. One can show (see Section 3.4.1 in [12]) that, given two different sets of linearly independent functionals $a_{1}, \ldots, a_{n} \in F^{\prime}$ and $\widehat{a}_{1}, \ldots, \widehat{a}_{n} \in F^{\prime}$ with $\mathcal{S}:=$ 
$\mathcal{S}_{a_{1}, \ldots, a_{n}}(F)=\mathcal{S}_{\widehat{a}_{1}, \ldots, \widehat{a}_{n}}(F)$, the corresponding maps $\sigma$ and $\widehat{\sigma}$ are gauge equivalent (i.e. there is an invertible $A \in \mathcal{M}_{n, n}(\mathbb{C})$ such that $\sigma(T)=A^{-1} \widehat{\sigma}(T) A$ for all $\left.T \in \mathcal{S}\right)$.

3.2. Resulting solution formulas. The following result says that if $U$ is a solution of the ncKP (1) and we can arrange $U \in \mathcal{S}_{a_{1}, \ldots, a_{n}}(F)$, then applying $\sigma$ to $U$ yields a solution of the matrix-KP (1) with values in the $n \times n$-matrices.

Proposition 3.4. Let $F$ be a Banach space and $a_{1}, \ldots, a_{n} \in F^{\prime}$ constant, linearly independent functionals. If $U=U(x, y, t) \in \mathcal{S}_{a_{1}, \ldots, a_{n}}(F)$ solves the $n c K P$, then $u(x, y, t)=$ $\sigma(U(x, y, t))$ solves the matrix-KP.

Proof. Since $U \in \mathcal{S}_{a_{1}, \ldots, a_{n}}(F)$, we can read the ncKP (1) as an operator equation in the Banach algebra $\mathcal{S}_{a_{1}, \ldots, a_{n}}(F)$. But $\sigma$ is an algebra homomorphism, so application of $\sigma$ to (1) immediately shows that $u=\sigma(U)$, which is a function with values in $n \times n$ matrices, again solves (1).

We are now in the position to prove Theorem 1.2.

Proof of Theorem 1.2.

Step 1: The operator-function $U=(I+M L)^{-1}(B-M A L)$ solves the ncKP (1) by Theorem 1.1. Thus, this is also the case for

$$
\begin{aligned}
\widetilde{U} & =U-B=(I+M L)^{-1}((B-M A L)-(I+M L) B) \\
& =-(I+M L)^{-1} M(A L+L B) .
\end{aligned}
$$

Step 2: As a consequence of the $n$-dimensionality condition (3),

$$
\begin{aligned}
\widetilde{U} & =-(I+M L)^{-1} M \hat{L}(A C+C B) \\
& =-(I+M L)^{-1} M \hat{L} \sum_{j=1}^{n} a_{j} \otimes c_{j}=\sum_{j=1}^{n} a_{j} \otimes f_{j} \in \mathcal{S}_{a_{1}, \ldots, a_{n}}(F),
\end{aligned}
$$

where $f_{j}=-(I+M L)^{-1} M \hat{L} c_{j} \in F$. Thus the operator-solution has the right form to apply Proposition 3.4, and we get a matrix-solution $u$ of (1) by

$$
u=\left(\left\langle f_{j}, a_{i}\right\rangle\right)_{i, j=1}^{n}
$$

Step 3: It remains to verify the solution formula in terms of the determinant available on the underlying quasi-Banach ideal. To this end we use the multiplicity property of determinants and the fact that on the finite-dimensional operators the generalized determinant $\delta$ coincides with the standard determinant det (in particular $\delta(I+a \otimes c)=$ $\operatorname{det}(I+a \otimes c)=1+\langle c, a\rangle$ for one-dimensional endomorphisms $a \otimes c)$. Thus,

$$
\begin{aligned}
\left\langle f_{j}, a_{i}\right\rangle & =1-\delta\left(I-a_{i} \otimes f_{j}\right) \\
& =1-\delta\left(I+(I+M L)^{-1}\left(a_{i} \otimes M \ell_{j}\right)\right) \\
& =1-\delta\left((I+M L)^{-1}\left(I+M L+a_{i} \otimes M \ell_{j}\right)\right) \\
& =1-\frac{\delta\left(I+M L+a_{i} \otimes M \ell_{j}\right)}{\delta(I+M L)} .
\end{aligned}
$$


REMARK 3.5. If in addition $D \in \mathcal{A}(E, F)$, then the following reformulation of the solution formula holds:

$$
u=\left(1-\delta\left(\begin{array}{cc}
I & -\left(L+a_{i} \otimes \ell_{j}\right) \\
M & I
\end{array}\right) / \delta\left(\begin{array}{cc}
I & -L \\
M & I
\end{array}\right)\right)_{i, j=1}^{n}
$$

Indeed, we check

$$
\begin{gathered}
\delta\left(\begin{array}{cc}
I & -L-a_{i} \otimes \ell_{j} \\
M & I
\end{array}\right) / \delta\left(\begin{array}{cc}
I & -L \\
M & I
\end{array}\right)=\delta\left(\left(\begin{array}{cc}
I & -L \\
M & I
\end{array}\right)^{-1}\left(\begin{array}{cc}
I & -L-a_{i} \otimes \ell_{j} \\
M & I
\end{array}\right)\right) \\
=\delta\left(\begin{array}{cc}
I & -(I+L M)^{-1}\left(a_{i} \otimes \ell_{j}\right) \\
0 I+(I+M L)^{-1} M\left(a_{i} \otimes \ell_{j}\right)
\end{array}\right) \\
=\delta\left(I-\left(0, a_{i}\right) \otimes\left((I+L M)^{-1} \ell_{j},-(I+M L)^{-1} M \ell_{j}\right)\right) \\
=1+\left\langle(I+M L)^{-1} M \ell_{j}, a_{i}\right\rangle=1-\left\langle f_{j}, a_{i}\right\rangle .
\end{gathered}
$$

Note that for the above calculation we have also used that $(I+M L)$ is invertible if and only if $(I+L M)$ is invertible and that

$$
\left(\begin{array}{cc}
I & -L \\
M & I
\end{array}\right)^{-1}=\left(\begin{array}{cc}
(I+L M)^{-1} & 0 \\
0 & (I+M L)^{-1}
\end{array}\right)\left(\begin{array}{cc}
I & L \\
-M & I
\end{array}\right) .
$$

4. Simplified formulas for the scalar KP equations. In the application of our solution formulas, involved computations are mainly caused by large determinants. The formula for the $n \times n$ matrix-KPs in Theorem 1.2 requires the calculation of $n^{2}+1$ determinants. For $n=1$ we may rewrite the formula so that only one determinant is left, resulting in a formula for which it becomes obvious that it generalizes the scalar one-soliton.

THEOREM 4.1. Let $E$ and $F$ be Banach spaces and $\mathcal{A}$ a quasi-Banach ideal equipped with a continuous determinant $\delta$. Let $A \in \mathcal{L}(E), B \in \mathcal{L}(F), D \in \mathcal{L}(E, F)$ and assume that $C \in \mathcal{A}(F, E)$ satisfies the one-dimensionality condition $A C+C B=a \otimes c$ where $a \in F^{\prime}$, $c \in E$.

Then

$$
u=-\partial_{x} \log \delta(I+L M),
$$

where $L(x, y, t)=\exp \left(A x+\alpha^{-1} A^{2} y-4 A^{3} t\right) C$ and $M(x, y, t)=\exp \left(B x-\alpha^{-1} B^{2} y-\right.$ $\left.4 B^{3} t\right) D$ is a solution of the KP equation

$$
\left(u_{t}-6\left(u_{x}\right)^{2}+u_{x x x}\right)_{x}+3 \alpha^{2} u_{y y}=0
$$

on $\{(x, y, t) \in \mathbb{R} \mid \delta(I+L M) \neq 0\}$.

Proof. Starting from (12) in the scalar case $n=1$ we use a different argument to reformulate the solution formula in terms of determinants. Since on the onedimensional operators the (usual) trace tr coincides with evaluation on the involved functional,

$$
u=\langle f, a\rangle=\operatorname{tr}(a \otimes f)=-\operatorname{tr}\left((I+M L)^{-1} M \hat{L}(a \otimes c)\right)
$$


Reinserting the one-dimensionality condition, we get

$$
\begin{aligned}
u & =-\operatorname{tr}\left((I+M L)^{-1} M(A L+L B)\right) \\
& =-\operatorname{tr}\left(M(I+L M)^{-1}(A L+L B)\right) .
\end{aligned}
$$

In the subsequent manipulations, we might deal with operators which not necessarily have finite rank. Thus we cannot use the trace tr for these arguments. For this reason we rewrite the formula in terms of the generalized trace $\tau$ associated to the determinant $\delta$ on the underlying quasi-Banach ideal using the fact that on the finite-dimensional operators both traces coincide.

$$
\begin{aligned}
u & =-\tau\left(M(I+L M)^{-1}(A L+L B)\right) \\
& =-\tau\left((I+L M)^{-1}(A L+L B) M\right) \\
& =-\tau\left((I+L M)^{-1}(L M)_{x}\right) \\
& =-\partial_{x} \log \delta(I+L M) .
\end{aligned}
$$

For details and references on the employed theory of traces and determinants on quasi-Banach ideals we refer to [12].

Acknowledgement. It is a pleasure to thank Professor Alexander Sakhnovich for inspiring discussions on the topic.

\section{REFERENCES}

1. H. Blohm, Solution of nonlinear equations by trace methods. Nonlinearity 13 (2000), $1925-1964$.

2. F. Calogero and A. Degasperis, New integrable PDEs of boomeronic type, J. Phys. A 39 (2006), 8349-8376.

3. B. Carl and C. Schiebold, Nonlinear equations in soliton physics and operator ideals, Nonlinearity 12 (1999), 333-364.

4. B. Carl and C. Schiebold, Ein direkter Ansatz zur Untersuchung von Solitonengleichungen, Jber. d. Dt. Math.-Verein. 102 (2000), 102-148.

5. A. Dimakis and F. Müller-Hoissen, With a Cole-Hopff transformation to solutions of the non-commutative KP hierarchy in terms of Wronski matrices, J. Phys. A 40 (2007), F321-F329.

6. A. Dimakis and F. Müller-Hoissen, Weakly nonassociative algebras, Riccati and KP hierarchies, Preprint 2007, see arXiv:nlin/0701010v4, 16 pp.

7. C. R. Gilson and J. J. C. Nimmo, On a direct approach to quasideterminant solutions of the noncommutative KP equation, J. Phys. A 40 (2007), 3839-3850.

8. M. Hamanaka, Notes on exact multi-soliton solutions of noncommutative integrable hierarchies, J. High Energy Phys. 02 (2007) 094.

9. B. A. Kupershmidt, KP or mKP. Noncommutative Mathematics of Lagarangian, Hamiltonian, and Integrable Systems. Math. Surv. Monogr. 78 (2000), American Mathematical Society, Providence, RI, 600 pp.

10. A. L. Sakhnovich, Matrix Kadomtsev-Petviashvili equation: matrix identities and explicit non-singular solutions, J. Phys. A 36 (2003), 5023-5033.

11. C. Schiebold, From the non-abelian to the scalar two-dimensional Toda lattice, Glasgow Math. J. 47A (2005), 177-189.

12. C. Schiebold, Integrable systems and operator equations, Habilitation Thesis (FriedrichSchiller Universität Jena 2004).

13. P. J. Olver and V. V. Sokolov, Integrable evolution equations on associative algebras, Comm. Math. Phys. 193 (1998), 245-268.

14. M. Wadati and N. Wang, Exact multi-line soliton solutions of noncommutative KP equation, J. Phys. Soc. Jpn. 72 (2003), 1881-1888. 